\title{
Transoral Robotic Surgery for Oropharyngeal Cancer
}

\author{
Vinidh Paleri ${ }^{a}$ Hannah Fox ${ }^{b}$ Stuart Winter ${ }^{c}$ \\ ${ }^{a}$ Royal Marsden Hospital, London, UK; ${ }^{b}$ Freeman Hospital, Newcastle, UK; \\ cOxford University Hospitals NHS Trust, Oxford, UK
}

\section{Keywords}

Oropharyngeal cancer · Robotic surgery $\cdot$ Transoral route

\begin{abstract}
Transoral robotic surgery has shown significant promise in the management of oropharyngeal cancer since its description in 2007. The oncological efficacy of this procedure has been proven in several single-centre studies, multicentre collaborative publications and systematic reviews. The rapid take-up of transoral robotic surgery shows greater acceptance by professionals and is associated with a relatively short learning curve. This overview discusses the rationale and principles underlying the use of transoral robotic surgery in primary and recurrent oropharyngeal cancer and the emerging role for this technique in diagnosing the unknown primary site, and summarizes ongoing research in this field.

(C) 2018 S. Karger AG, Basel
\end{abstract}

\section{Introduction}

Transoral robotic surgery (TORS) was initially conceived for oropharyngeal cancer. The traditional treatment for this primary site, until the turn of the millennium, had been open surgery; however, reports suggesting lack of benefit from open surgery [1] and randomized trials reporting on non-surgical management (without a surgical comparative arm) led to primary open surgery being abandoned as a treatment modality for oropharyngeal cancer. The emergence of transoral laser microsurgery (TLM) offered the prospect of a surgical procedure with less morbidity. Publication of several reports of good oncological control with 
Paleri et al.: TORS for Oropharyngeal Cancer

Table 1. Prevalence (\%) of HPV-positive oropharyngeal cancers around the world [4]

\begin{tabular}{lllll}
\hline & Before 1995 & $1995-1999$ & $2000-2004$ & $2005-2015$ \\
\hline Worldwide & $32.3(46.7-56.9)$ & $37.0(30.1-44.0)$ & $51.8(46.7-56.9)$ & $52.9(42.8-63.0)$ \\
North America & $43.1(28.8-57.4)$ & $47.3(31.3-63.3)$ & $66.6(59.0-74.2)$ & $64.7(48.0-81.4)$ \\
Europe & $28.0(18.2-37.7)$ & $35.6(26.5-44.7)$ & $42.4(35.6-49.1)$ & $49.5(36.4-62.6)$ \\
\hline
\end{tabular}

Table 2. Prevalence (\%) of HPV-positive oropharyngeal tumours in the UK [2]

\begin{tabular}{|c|c|c|c|c|c|c|c|c|c|c|}
\hline & 2002 & 2003 & 2004 & 2005 & 2006 & 2007 & 2008 & 2009 & 2010 & 2011 \\
\hline 1 & 50.0 & 48.1 & 52.6 & 54.1 & 47.4 & 55.4 & 54.2 & 53.7 & 53.1 & 49.1 \\
\hline
\end{tabular}

TLM combined with low morbidity led to interest in primary surgery for this site being rejuvenated. While TLM has shown undoubted oncological efficacy in this setting, most work on TLM primarily pertained to tumours of the tonsil. Due to difficulties with resecting cancers of the tongue base using an endoscope, a microscope and co-axially mounted laser, the tongue base continued to be an orphan site with very few reports presenting data on TLM outcomes for this subsite. Even for tonsil cancers, surgeons and multidisciplinary teams (MDTs) were slow to adopt TLM for several reasons. The technique is difficult to teach which resulted in a long learning curve. TLM involves cutting through the tumour to identify its depth, with further sampling of the defect to confirm "clear" margins; thus, pathological margin assessment of the transected specimen was beset with controversies and frequently resulted in patients receiving double or triple modality treatment.

The manoeuvrability and optics of a robotic system, the prospect of en bloc resection and rapid adoption of robotic technology at other primary sites were attractive to head and neck surgeons. TORS offered the optimal balance of transoral access with limited morbidity, en bloc resection and a more rapid learning curve.

This review will address the rationale and evidence base supporting TORS for primary and recurrent cancers of the tonsil and tongue base, and the emerging role of TORS in the investigation of the unknown primary tumour. This work will also briefly discuss the technique of TORS for oropharyngeal tumours, pathological assessment and the trials that are ongoing currently in this field.

\section{Epidemiology of Oropharyngeal Cancer}

The epidemiological trends with respect to the rising incidence of human papillomavirus (HPV)-related cancers at this primary site has been a key driving force in transoral surgery for oropharyngeal cancer. Currently, good epidemiological data exist for increased numbers of HPV-related oropharyngeal cancer around the world [2,3] (Table 1). While there has been a continued increase in the prevalence of HPV oropharyngeal cancer over time a recent UK study suggests this has plateaued (Table 2). This cancer is seen primarily in younger men in their fifties [3], typically with less comorbidity and thus following treatment they are likely to survive longer. Therefore, in addition to oncological outcomes, improved function and reduced toxicity have become key objectives in treating this group of patients. 
Paleri et al.: TORS for Oropharyngeal Cancer

\section{Rationale for Incorporating Surgery into the Treatment of Oropharyngeal Malignancies}

\section{HPV-Related Squamous Cell Cancers}

Several publications have confirmed that radical doses of chemoradiotherapy (CRT) used to treat head and neck cancer (with the aim of organ preservation) cause significant morbidity for patients in the short and long term. While the use of radiotherapy with concomitant chemotherapy improves the survival and locoregional control when compared with radiotherapy alone [5, 6], it also increases the burden of acute toxicity [7]. Mucositis is one of the main acute symptoms: Trotti et al. [8] demonstrated, in a systematic literature review including data from of 6,181 patients, that not only did $80 \%$ suffer this side effect, but it also led to hospitalization in $16 \%$ and modification of radiotherapy regimes in $11 \%$. The rate of severe mucositis was higher with the use of altered fractionation regimes as compared to conventional radiotherapy. It is now understood that these acute toxicities persist into the long term, although this is often underreported or underestimated in trial reports [9]. Machtay et al. [10] performed a meta-analysis of 230 patients included in 3 large trials (RTOG 91-11, RTOG 97-03 and RTOG 99-14) to investigate whether they continued to experience toxicity more than 180 days following treatment. Indeed, $43 \%$ of patients were found to still be experiencing grade 3 or higher toxicity, were continuing to use a gastrostomy for feeding or had died from a cause thought to be secondary to laryngeal dysfunction. Those with higher $\mathrm{T}$ stage, higher age or a primary tumour in the larynx or pharynx were found to be most at risk of long-term toxicity. Other authors have echoed the finding of long-term dysphagia after CRT [11] and many patients require a permanent gastrostomy or nasogastric tube [12,13]. Even with parotid-sparing intensity-modulated radiation therapy (IMRT) [14], one third of the patients experience grade 2 or worse xerostomia as assessed by the Late Effects of Normal Tissues Subjective-Objective Management Analytic measure. These long-term functional problems such as effects on swallow and dry mouth cannot be ignored; indeed, the functional outcome of treatment is considered by many to hold primary importance when making treatment decisions [15].

As this cancer in this relatively younger patient group responds well with either treatment modality, survivorship issues become important. In patients receiving CRT, top priorities at various time points are consistently xerostomia and dysphagia [11], the former being a side effect seen with radiation therapy alone. Prospective observational studies suggest that both side effects can be abrogated with a primary TORS approach. In an observational study [16] of 74 patients treated with TORS and adjuvant therapy and 46 patients treated with definitive CRT, primary TORS resulted in significantly better saliva-related quality of life than definitive CRT at $1,6,12$, and 24 months ( $p<0.001, p=0.035, p=0.005, p=0.007)$. Using gastrostomy use as a surrogate marker of poor swallowing, in a retrospective matched cohort study, Sharma et al. [17] identified that patients who received TORS-based therapy had lower gastrostomy tube prevalence after treatment (risk reduction by $57 \%$ ); over time, gastrostomy prevalence decreased for both groups, with $3 \%$ of gastrostomy patients in the TORS group and $11 \%$ in the non-surgical group. Similar findings were identified in a systematic review involving 20 case series, including 8 IMRT studies (1,287 patients) and 12 TORS studies $(772$ patients). While the oncological outcomes were comparable, the adverse events profile was different: oesophageal stenosis $(4.8 \%)$ and osteoradionecrosis $(2.6 \%)$ for IMRT, haemorrhage $(2.4 \%)$ and fistula $(2.5 \%)$ for TORS. Incorporation of TORS into the treatment allows clinicians to tailor adjuvant therapy in patients who otherwise would receive CRT. In a study of 76 patients who underwent TORS, Gildener-Leapman et al. [18] found that up-front TORS de-intensified adjuvant therapy in $76 \%$ of stage I/II and $46 \%$ of stage III/IV patients who avoided CRT. Conversely, the pathological staging from TORS resulted in $33 \%$ of patients who 
Paleri et al.: TORS for Oropharyngeal Cancer

would have received radiotherapy alone based on clinical staging, to be intensified to receive adjuvant CRT.

\section{Non-HPV-Related Squamous Cell Cancers}

These cancers typically have a worse prognosis than HPV-positive squamous cell carcinoma. All level 1 evidence for this group of patients is for non-surgical treatment. The French 94-01 phase III multicentre randomized trial recruited 226 patients, comparing radiotherapy alone with radiotherapy and concomitant chemotherapy. Although explicit HPV testing was not done, the patient cohort with large primary sites and small nodal disease is indicative of HPV-negative tumours. They reported 5-year overall survival (22.4 vs. 15.8\% $[p=0.05]$ ) and locoregional control ( 47.6 vs. $24.7 \%$ [ $p=0.002]$ ) favouring the latter arm. The RTOG 1221 was set up to compare primary transoral surgery (TORS or TLM) with adjuvant radiation against primary radiation with concomitant chemotherapy. Owing to poor accrual, this trial had to be closed. Other prospective data sets where HPV-negative cancers have been treated by TORS and adjuvant radiation indicate high control rates, from 80 to 94\% [19-21]; however, the selection bias in these studies is inevitable. A UK-led randomized trial for this group of patients with a surgical arm is ongoing (CompARE; UKCRN 18621).

A few prospective series have identified that adding surgery to the treatment for patients with HPV-negative cancers offers better control rates [22]. As discussed above, the morbidity and mortality rates attributed to open surgery [1] do not apply to surgical practices in the current day, and especially to transoral surgery; data on 30-day mortality from the UK national head and neck database indicates a mortality of $<1 \%$ [23].

\section{Non-Squamous Cancers}

Minor salivary gland tumours are the most common non-squamous cancers seen in this anatomical site. The primary management is surgery. Following careful assessment, if access is found to be optimal, as is often the case with these tumours, transoral resection with or without adjuvant radiation offers equivalent control rates with less disruption of anatomy, and thus quicker recovery [24].

\section{TORS in Tonsil and Tongue Base Cancers}

\section{Tonsil Cancer}

Transoral laser surgery has been shown to be effective in the management of tonsil tumours. The principles of this involve resection of the tumour just outside the tumour capsule. Assessing the depth of the tumour is achieved frequently by transecting the tumour until the "normal" tissues are reached. The cutting characteristics of the laser mean this is clearly demonstrable. However, margins on the specimen are frequently reported as close and marginal biopsies are therefore used to assess status. While this approach is oncologically safe, it can present practical concerns in deciding whether adjuvant therapy is needed [25]. Nevertheless, the significant amount of work in the TLM literature has paved the way to establish TORS for tonsil tumours as bona fide management with firm long-term oncological outcomes.

The principle of TORS surgery is en bloc resection with a clear margin. The foundations for en bloc resection of tonsil cancers via a transoral route had been laid back in the 1950s when Huet [26] described this procedure. This involved transoral resection of the tonsil and the superior constrictor muscle deep to the parapharyngeal space. Holsinger et al. [27] reported good outcomes for tonsil tumours with this technique. En bloc resections of the tonsil, although feasible with the TLM approach, are not easy due to the line of sight working 
Paleri et al.: TORS for Oropharyngeal Cancer

and instrumentation. The advent of TORS has allowed surgeons to perform en bloc resections at this primary site. The superior optics and manoeuvrability combined with the ergonomics have meant that the learning curve for transoral robotic radical tonsillectomy is not as steep as that of TLM.

TORS radical tonsillectomy is one of the first procedures that is undertaken by surgeons in the early stages of their TORS practice. The first report of TORS radical tonsillectomy was published in 2007 by Weinstein et al. [28]. This included a cohort of 27 adult patients with tonsil tumours staged T1-T3. Weinstein etal. reported a negative margin of $93 \%, 0 \%$ mortality and an acceptable complication rate with 4\% gastrostomy dependence at 6 months. Similar high control rates have been replicated in several single-centre series; a multicentre review and a systematic review have confirmed the oncological basis for TORS radical tonsillectomy. A global multi-institutional study [19] that collated outcomes for 410 patients from 11 centres demonstrated 2-year locoregional control rates of $92 \%$ and disease-free survival of $91 \%$. A meta-analysis [29] comparing TORS, neck dissection and adjuvant therapy as appropriate with IMRT (with or without chemotherapy) and salvage surgery as appropriate, identified 20 case series. The data from 772 surgical patients were compared with 1,287 patients receiving primary non-surgical treatment. While oncological outcomes were comparable (2-year overall survival estimates ranged from 84 to $96 \%$ for IMRT and from 82 to $94 \%$ for TORS), the main difference lay in the adverse effect profiles. Patients who received IMRT experienced oesophageal stenosis (4.8\%), osteoradionecrosis (2.6\%) and gastrostomy tubes (43\%), while those who received primary surgery experienced haemorrhage $(2.4 \%)$, fistula $(2.5 \%)$, and gastrostomy tubes at the time of surgery (1.4\%) or during adjuvant treatment (30\%). Tracheostomy tubes were needed in $12 \%$ of patients at the time of surgery, but most were decannulated prior to discharge. TORS is currently used and approved by the FDA for T1 and T2 cancers in the USA. The multi-institutional series also reflected the data from other publications that TORS is performed primarily for these stages, although a small proportion of T3 and T4 tumours were also included.

The aim of resecting tonsil tumours with TORS is to ensure compartmental resection of the tonsil, the constrictor bed it sits on along with a cuff of soft palate and the tongue base to ensure histologically negative margins. The technique of this procedure is well described [28], but these are the key steps involved: incision through the pterygomandibular raphe to identify the parapharyngeal space with the help of the medial pterygoid tendon, separating the constrictor muscles from the parapharyngeal fat, mobilizing the superior pole of the tonsil with an adequate cuff of tissue from the soft palate, progressive dissection in the parapharyngeal space to free the stylopharyngeus and pharyngeal constrictors, mobilizing tongue base mucosa and deep tissue between the anterior and posterior tonsillar pillars, resecting at least a centimetre segment of styloglossus to ensure a histologically negative margin, making index cuts on the posterior pharyngeal wall to avoid resection of excessive amounts of mucosa and progressive transection of the constrictor muscle along with the stylopharyngeal musculature to achieve an en bloc resection. While some authors recommend suturing the posterior pharyngeal wall to the posterior aspect of the soft palate to avoid nasal regurgitation, we have not specifically found this to be an issue. Postoperatively the tonsil defect (Fig. 1) is left to heal on its own and complete mucosalization normally takes place within 6 weeks. Patients commence oral feeds much before this time and usually are free of nasogastric feeds within days [30].

\section{Tongue Base Cancers}

TORS is used for T1 and T2 tongue base cancers, but given the relative rarity of these tumours, the bulk of published data includes this site along with tonsil tumours. The principles of the operation are similar to tonsil tumours where an en bloc resection is performed. 
Fig. 1. Clinical photograph of the defect following lateral oropharyngectomy.

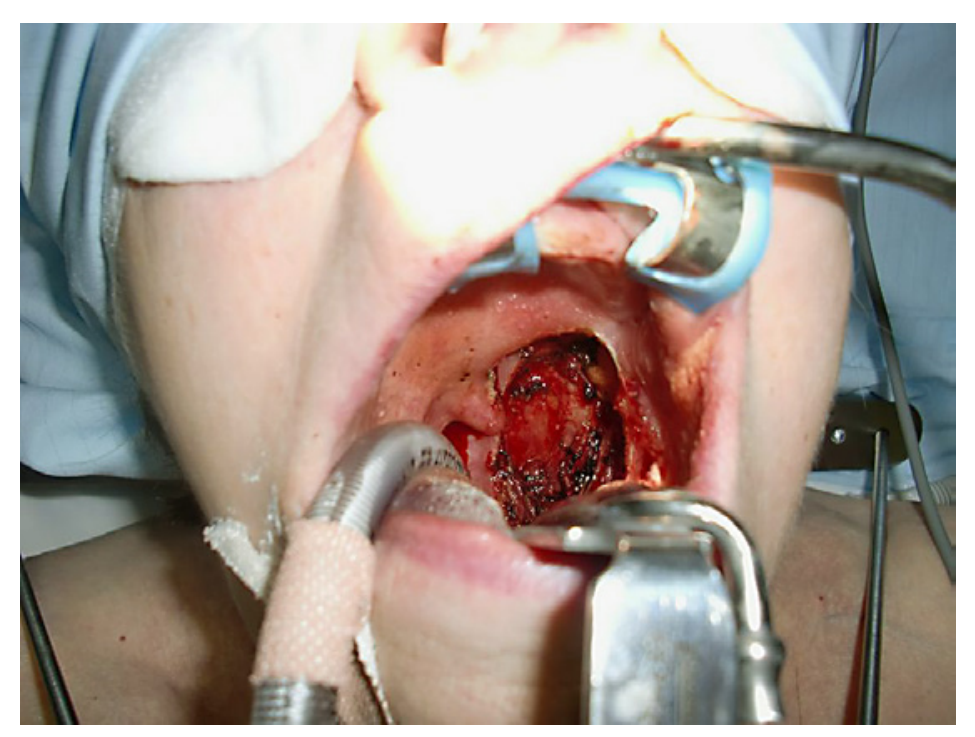

The tumour on the tongue base is exposed using relevant retractors, the choice of which is based on the patient's anatomy and ease of access. Careful assessment of the pre-operative scans is important to assess the precise location and lateralization of the tumour. Tumours crossing the midline are not good candidates for TORS. The operation commences with an incision along the posterior one third of the tongue. This is deepened followed by a midline cut towards the vallecula. The vallecula identifies the depth of the resection needed, and the lateral margin is established. This may involve part of the lower third of the tonsil. With progressive dissection, the lingual artery will be encountered. The bedside assistant can control bleeding from this vessel with ligaclips applied transorally. If the defect needs to be sampled, this is inked on table prior to excision to allow differentiation between the superficial and deep surfaces, and orientated for the pathologist (see the section "Margin Assessment of the Primary Tumour" below). Our practice is to place a nasogastric tube routinely in all patients; the timing of removal is dependent on the recovery of swallowing and is judged by the rehabilitation experts.

\section{Margin Assessment of the Primary Tumour}

Achieving tumour-free margins must be the goal for primary surgery, as positive margins correlate with local failure, the latter being the greatest single cause of mortality in head and neck cancer patients. The status of the margins dictates adjuvant therapy and ultimately whether a surgical procedure is deemed a success by both patients and clinicians. The importance of a coherent approach to margin assessment and management by individual MDTs cannot be overemphasized.

There are two approaches for the assessment of margins in transoral surgery - "specimen-driven" and "defect-driven." A defect-driven approach has been used widely in transoral laser surgery for non-glottic cancers, where the ability to define the interface between tumour tissue and normal tissue permits tailored resection of the tumour and can be followed by a sampling of the tumour bed. However, this can lead to variable practices within MDTs when determining whether adjuvant therapy is required. In TORS, the superior access and manoeuvrability allow for en bloc resection and a more comprehensive evaluation of the margins within the defect following specimen resection. It is generally possible to sample the whole resection bed, frequently to a depth of several millimetres or more. A combination of 
a "specimen-driven" and "defect-driven" approach enables greater confidence that clear margins have been achieved. However, there will still be areas where anatomical structures and boundaries limit the ability to resect a wide margin, and clear communication and clarity within individual MDTs on how to manage these situations is critical.

Irrespective of which approach is used, close communication between surgeon and pathologist is essential.

Several methods have been described to achieve this, which will not be exhaustively discussed here. In centres where the pathology lab is on site, the tumour specimen is best sectioned by a surgeon or a pathologist with both specialists being present. Areas where any concern is identified about positive margins can be addressed at the same time on table. There are few data to support on-table frozen margins if the adequacy of resection is not in doubt; however, steps must be taken to ensure that the surgeon is convinced about the adequacy of the resection. If a positive margin is suspected, further resection can be performed on the same side until confidence and/or negative frozen margins are obtained. In centres where this service is not feasible, careful attention to specimen orientation is essential.

Our rationale and approach to mounting the specimen is as follows. Following a TORS resection, the en bloc specimen has a convex deep aspect to it. However, after formalin fixation, the convex deep margin that firmly abuts the specimen board is flattened out. During sectioning of the formalin-fixed specimen that has been mounted with the deep margin against the specimen board, the muscle layers that were seen to be freely mobile on the undersurface of the tumour after resection are compressed and distorted, leading to underestimation of the margins. We mount the specimen with the deeper side facing up, pinned to maintain the natural convexity of the resection (Fig. 2); this addresses the issues mentioned above. To ensure optimal communication, it is our practice to photograph and label all resections, which are then sent to the pathology team prior to specimen processing.

Following pathological analysis any areas of uncertainty are discussed by the pathologist and operating surgeon, as are any potentially positive margins. Our technique has evolved over time, and other centres may have equally successful methods. The key is clarity of communication and careful attention to the specimen and marginal biopsies.

\section{Management of the Neck}

In the setting of squamous cancers of the tonsil and tongue base, there are no scenarios where the neck will not need to be addressed. There remains some debate as to whether the tonsil and neck dissection should be performed at the same time or with the procedures staged. There are advantages and disadvantages to both approaches. Performing the tonsil resection and neck dissection at the same time allows for a single operative procedure, but with a small risk of creating a fistula into the neck. A staged operation, where the neck dissection is performed first reduces the risk of a fistula into the neck and allows for full use of the allocated robotic time that is available for surgical teams on specified days. This approach does necessitate that the two procedures are separated by days.

The risk of a fistula into the neck is also influenced by the levels of the neck that are dissected. No consensus exists, but many surgical teams will perform a level II-IV neck dissection for N1 and N2a necks, while others dissect level I-IV. The importance of addressing level I in this setting has yet to be elucidated, but dissecting this level increases the risk of a fistula in synchronous procedures. It is generally agreed that at the time of the neck dissection, individual branches of the external carotid artery (lingual, facial and ascending pharyngeal) are identified and individually ligated to reduce the risk of postoperative haemorrhage after transoral surgery [31, 32]. 


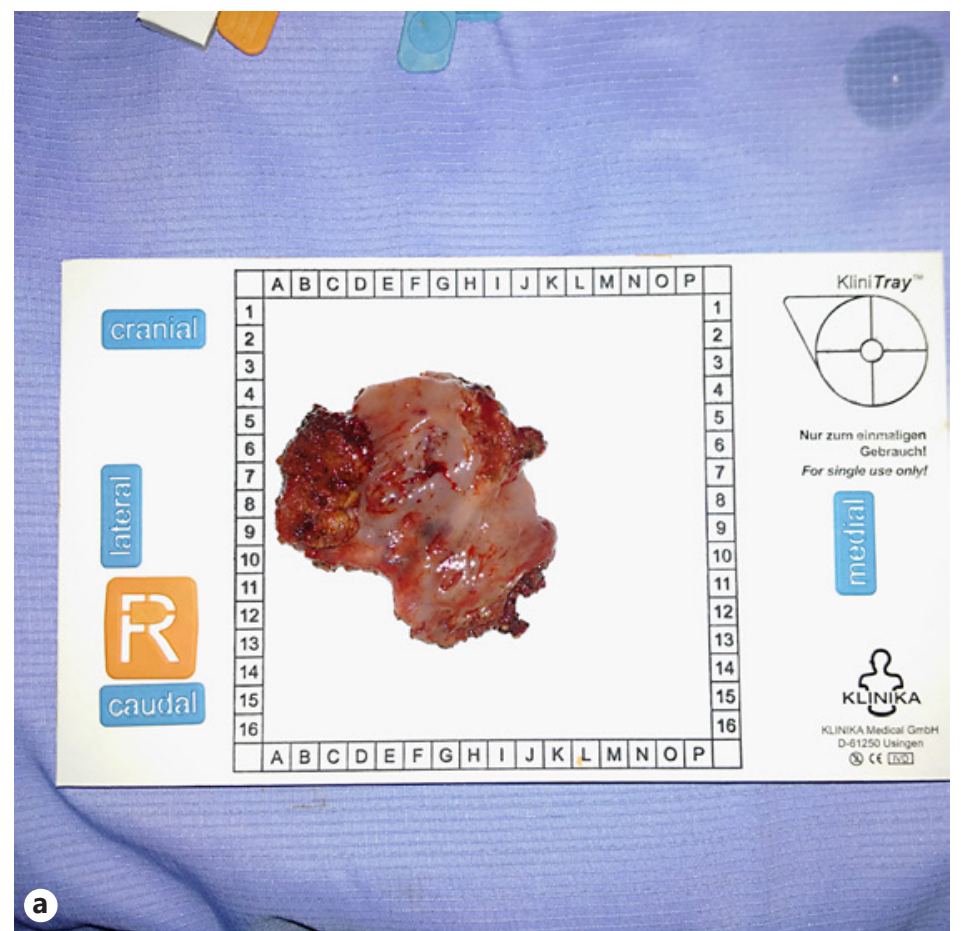

Fig. 2. Resected specimen is ori-

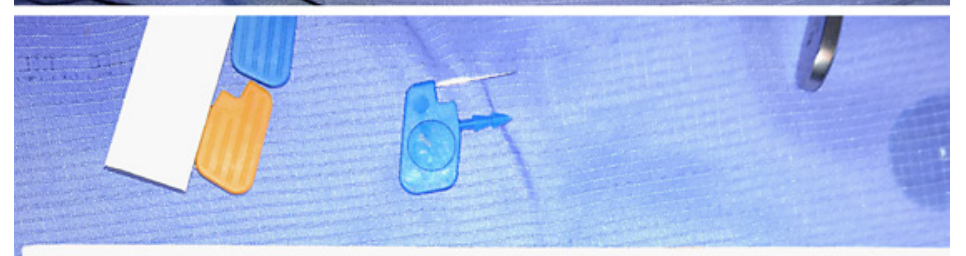
entated and mounted with the deeper side facing up, taking care not to distort the convexity.

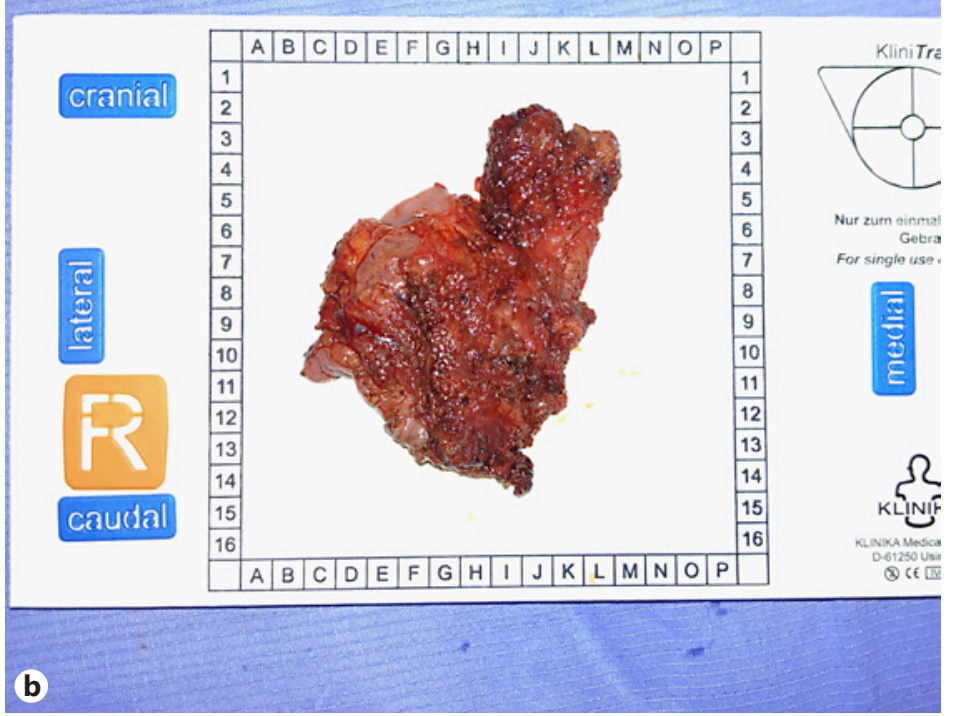

\section{Postoperative Adjuvant Treatment}

It is essential and mandatory that prior to setting up a transoral robotic surgical practice, institutional agreement and protocols for surgical and radiation oncology treatment are agreed for these patients. It is especially relevant in the setting of HPV-positive oropharyngeal cancer where significant controversy exists about the role of the surgery given the high onco- 
logical efficacy of radiation therapy or CRT in the cohorts being chosen for transoral surgical resection. It is imperative that treatment-related adverse effects, oncological and functional outcomes are collected in this group of patients. The authors strongly recommend that where ongoing trials are available patients be recruited into these trials.

Most patients will need nasogastric tube placement until their swallow function is improved. It is rare for patients to have a swallowing problem by the time they need adjuvant treatment [30].

Adjuvant radiation is based on the results of pathological examination, patient factors and institutional practice. In general, adjuvant radiation is delivered in the presence of positive margins and adverse features in the primary cancer or the neck dissection specimen [33]. However, several prospective data sets have questioned the value of concurrent CRT in the adjuvant setting for HPV-positive tumours [34, 35], and de-intensification regimes are being tested in ongoing trials.

\section{TORS for the Unknown Primary}

This is an exciting area for development, triggered by the first report by Mehta et al. [36] that has been replicated in other series. It is entirely conceivable that HPV-positive microcancers originate from the palatine and lingual tonsils. In the absence of any evidence of a primary site on conventional cross-sectional imaging, the tongue base is clinically assessed, and random biopsies are taken. Random biopsies will have a low probability of primary site detection given the large area of the tongue base. Tongue base mucosectomy has been designed to remove the entire lingual tonsils from the circumvallate papillae to the vallecula. This permits a full histological evaluation and further identification of the primary site.

The sensitivity and specificity of tongue base mucosectomy reflects in part the investigations that have been performed prior to the procedure, as these will have diagnosed or raised the suspicion for the site of the primary in several cases. A recent systematic review by Fu et al. [37] on identifying the unknown primary has reported on 8 studies with 139 patients. In the subset of patients in whom physical examination, direct visual examination and imaging were all unremarkable, $59 \%$ of primary tumours were identified. The majority were on the ipsilateral tongue base (96\%) while 6\% were in the contralateral tongue base. This series reports an overall $7 \%$ complication rate with postoperative bleeding being the most common complication. While not measured directly in this population, swallowing function recovers by 6 weeks in all patients who undergo TORS for primary cancer [30]; in the study by Fu et al. [37] only $1 \%$ did not return to a normal diet. It is yet to be demonstrated that identifying the primary tumour will alter survival and functional outcomes as adjuvant treatment can be tailored to the individual patient. However, the ability to treat a unilateral area may have significant effects on swallowing in the long term. The experience in the UK is broadly in line with the above studies. In a homogeneous cohort of 32 patients who were classified as cancer of unknown primary following clinical examination, cross-sectional imaging, PET-CT imaging, bilateral tonsillectomy and guided biopsies of the tongue base, Winter et al [38] report a 53\% primary site identification in the tongue base, with $12 \%$ on the contralateral tongue base.

The technique can be performed using any of the retractors used to expose the tongue base. Incisions are made through the full thickness of the tongue base mucosa up to the muscle layer, at the level of the circumvallate papillae. The plane between the submucosa and the muscle is developed, and the entire mucosal layer of the tongue base is systematically removed down to the vallecula. While it is feasible to remove the entire tongue base mucosa as a single specimen, the authors recommend a midline incision to remove the two sides separately as this reduces trauma to the specimen and allows easier orientation. 
Paleri et al.: TORS for Oropharyngeal Cancer

Table 3. Outcomes following TORS for recurrent cancer

\begin{tabular}{|c|c|c|c|c|c|c|c|}
\hline Author & $\begin{array}{l}\text { Patients, } \\
n\end{array}$ & $\begin{array}{l}\text { Proportion of } \\
\text { early T stages } \\
\text { (T1 and T2), \% }\end{array}$ & Outcomes (RFS) & $\begin{array}{l}\text { Mean specimen } \\
\text { size, } \mathrm{mm}\end{array}$ & $\begin{array}{l}\text { Operative } \\
\text { time, min }\end{array}$ & $\begin{array}{l}\text { Positive } \\
\text { margins, \% }\end{array}$ & $\begin{array}{l}\text { Blood } \\
\text { loss, } \mathrm{mL}\end{array}$ \\
\hline White et al. [43] & 64 & 92 & $74 \%$ at 2 years & n.a. & 111 & 9.0 & 49 \\
\hline Dabas et al. [44] & 30 & 89 & $57 \%$ at 19 months & n.a. & 62 & 6.7 & 23.3 \\
\hline Paleri et al. [40] & 18 & 89 & $63 \%$ at 2 years & $\begin{array}{l}28 \times 18 \times 6 \text { to } \\
62 \times 50 \times 15\end{array}$ & 97 & 11.1 & n.a. \\
\hline
\end{tabular}

RFS, recurrence-free survival; n.a., not available.

\section{Safety of TORS in Oropharyngeal Cancer}

Earlier concerns about safety of en bloc resection in these patients has been allayed by several publications. While these publications do not necessarily report oropharyngeal cancer cohorts in isolation, the overwhelming majority of patients have an oropharyngeal primary; thus, the data are applicable to this cohort. In a systematic review involving 12 TORS studies with 772 patients, haemorrhage was seen in $2.4 \%$ and fistula in $2.5 \%$. In a prospective review of 305 patients from the American College of Surgeons NSQIP data set, published since the systematic review above, Su et al. [39] identified a complication rate of $7.9 \%$ and a 30 -day mortality of $0.7 \%$. Increasing length of stay was mainly related to concurrent comorbidity.

\section{Recurrent Cancer}

The standard of care for recurrent oropharyngeal cancers is open resection. In selected cases, TORS has shown good oncological outcomes for this patient group. The published literature along with the unpublished series from the author's case load [40] is presented in Table 3. Significant expertise with TORS is needed prior to embarking on these procedures. It must be recognized that cross-sectional imaging, especially for the tongue base, is done with the tongue in the resting position in the mouth. During TORS, the tongue is brought out for access and the blades of the mouth retractor will distort tongue anatomy; thus, the depth of the tumour as seen on MRI may not be accurate, and in cases where the tumour is ill-defined, additional techniques will be needed to define the cancer extent on table. Adjuncts like intraoral ultrasound (delivered using robotic arms for access) will help define the tumour thickness and boundaries in these patients, as the interface between tumour tissue and normal tissue is not well defined. When well performed, significant specimen sizes can be resected with adequate resection margins. Patients will need prolonged rehabilitation given that the oropharynx is already compromised by the scarring and diminished function from previous CRT. Free flap reconstruction is not needed in most cases as the defect will heal by secondary intention. The authors experience correlates with published data that suggest that free flap reconstruction, independent of $T$ stage, has an adverse effect on functional outcomes. In cases where the resection is through into the neck or where the tissue is felt to be unhealthy, a TORS-assisted free flap inset is needed and can be performed. The neck should be dealt with as appropriate, and in cases where no neck disease is present, the relevant feeding vessels should be addressed. 
Fig. 3. Robotic assisted low inset of fasciocutaneous free flap after resection of posterior pharyngeal wall tumour.

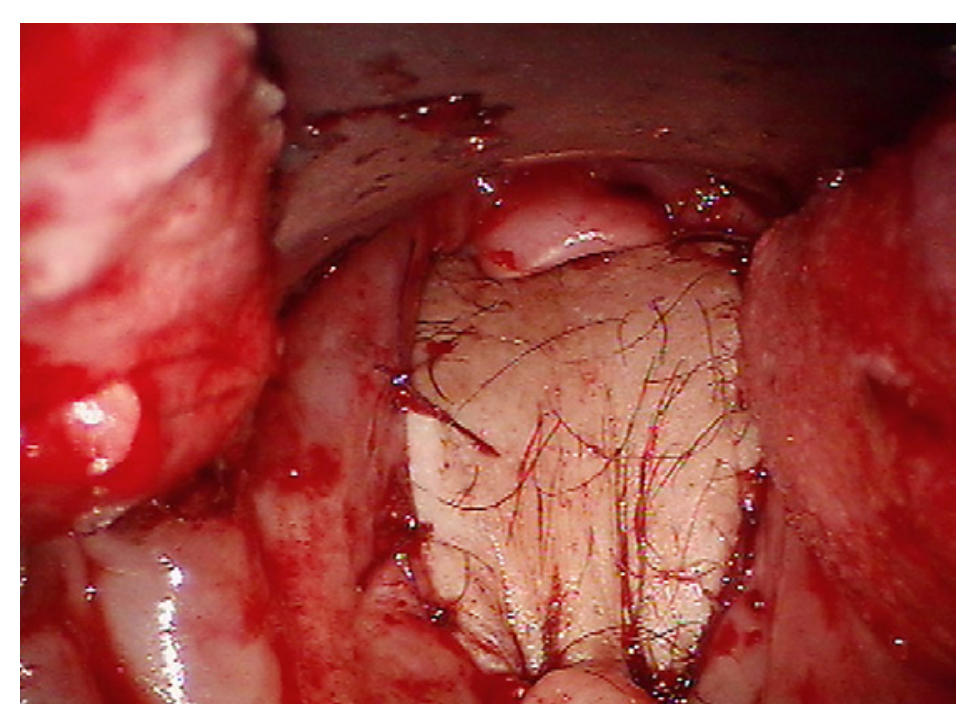

\section{Free Flap Inset via TORS}

TORS has allowed removal of complex tumours from the oropharynx. As acceptability of the recognition of the safety and efficacy of the procedure has evolved, larger and more complex tumours are being treated. This has increased the need to reconstruct the oropharynx to provide velopharyngeal competence and reduce fistula risk. A number of algorithms have been proposed depending on the site and size of the defect. These range from allowing healing by secondary intent to velopharyngoplasty with local flaps, local flaps alone or in larger defects regional and free flap reconstruction. Robotic-assisted mobilization and inset of the flaps have been described in extending the range of options for these patients (Fig. 3) $[41,42]$.

\section{Rehabilitation}

All patients, particularly those with recurrent cancer, need careful pre-operative speech and swallow assessment, and a multidisciplinary approach is mandatory for these patients. Appropriate feeding routes are decided based on this assessment. Speech and dysphagia rehabilitation vary significantly, and any intervention needs to be tailored to the individual's pre-existing dysfunction and the resultant postoperative dysfunction. This includes a combination of dysphagia-specific strategies for the rehabilitation process.

\section{Research in Transoral Surgery for Oropharyngeal Cancer}

The increased incidence, new techniques for surgical and non-surgical treatment and a younger patient population have created a need for robust evidence to be generated. Clinicians across the world have recognized this, and Table 4 summarizes the ongoing clinical trials on this topic. It is very likely that treatment processes will change for this disease in the next decade. 


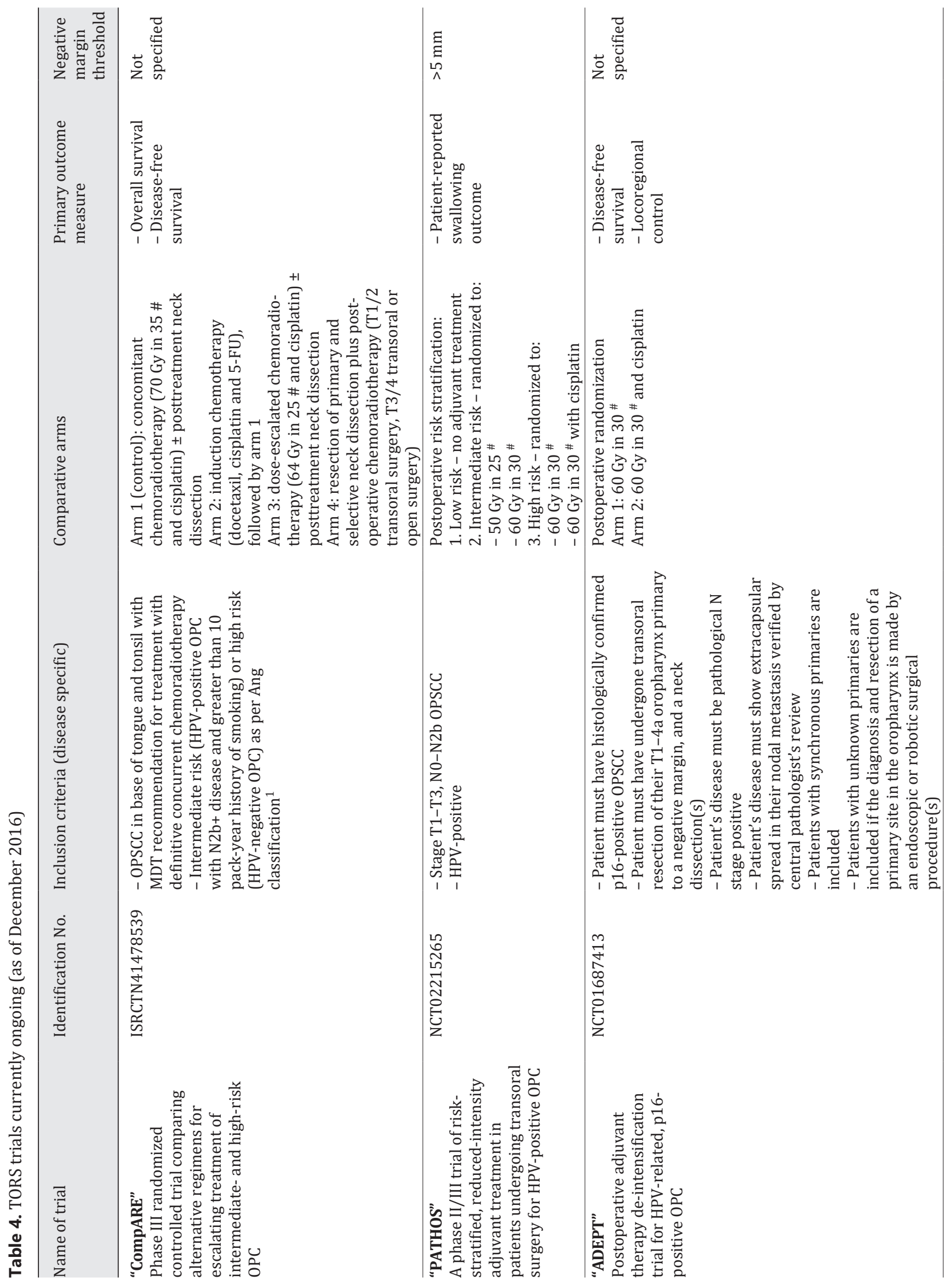



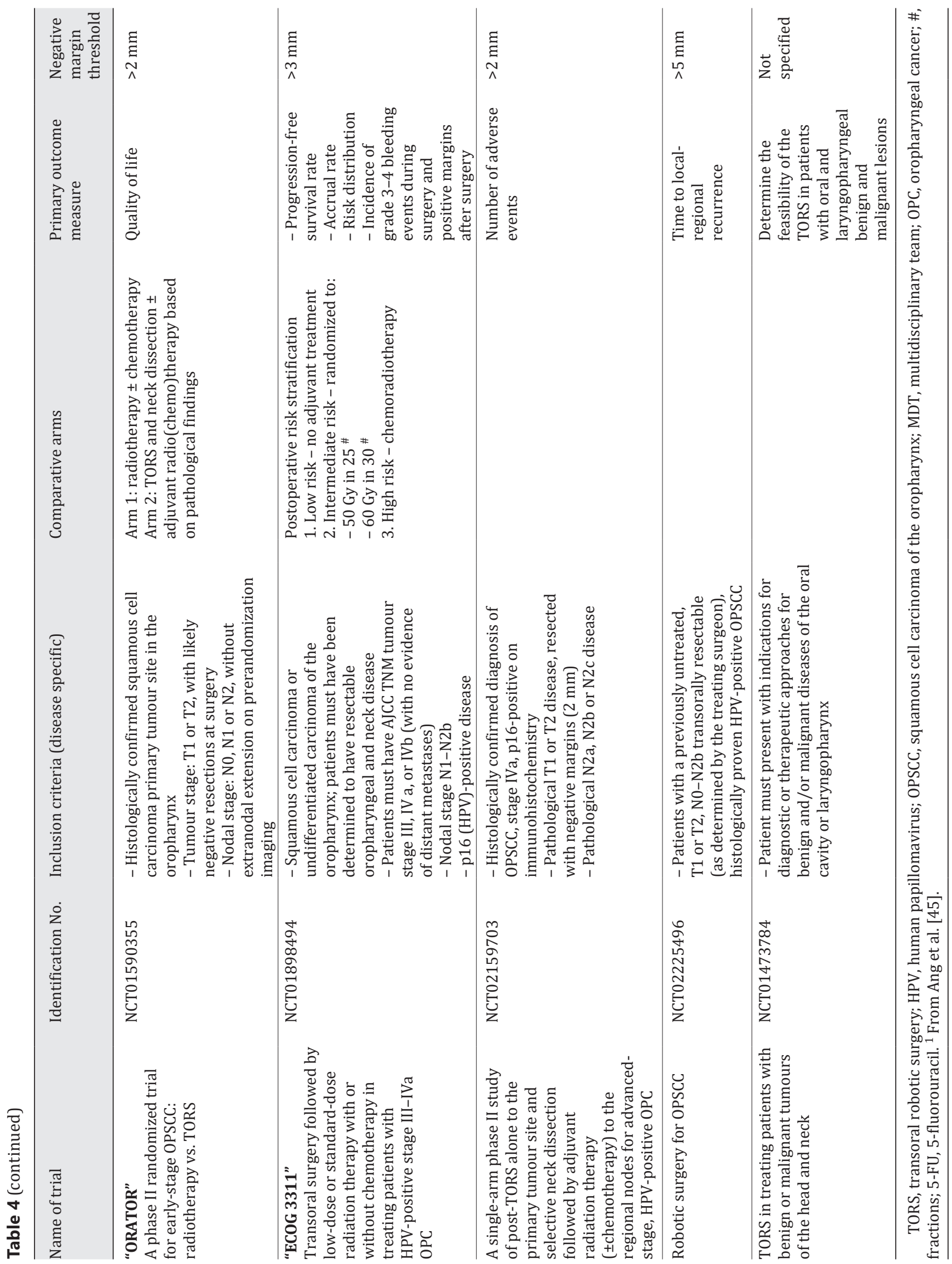
Paleri et al.: TORS for Oropharyngeal Cancer

\section{References}

1 Parsons JT, et al: Squamous cell carcinoma of the oropharynx: surgery, radiation therapy, or both. Cancer 2002;94:2967-2980.

2 Schache AG, et al: HPV-related oropharynx cancer in the United Kingdom: an evolution in the understanding of disease etiology. Cancer Res 2016;76:6598-6606.

3 Mehanna H, et al: Geographic variation in human papillomavirus-related oropharyngeal cancer: data from 4 multinational randomized trials. Head Neck 2016;38(suppl 1):E1863-E1869.

4 Stein AP, et al: Prevalence of human papillomavirus in oropharyngeal cancer: a systematic review. Cancer J 2015;21:138-146.

5 Bourhis J, et al: Individual patients' data meta-analyses in head and neck cancer. Curr Opin Oncol 2007;19: 188-194.

6 Pignon JP, et al: Meta-analysis of chemotherapy in head and neck cancer (MACH-NC): an update on 93 randomised trials and 17,346 patients. Radiother Oncol 2009;92:4-14.

7 Abendstein H, et al: Quality of life and head and neck cancer: a 5 year prospective study. Laryngoscope 2005; 115:2183-2192.

8 Trotti A, et al: Mucositis incidence, severity and associated outcomes in patients with head and neck cancer receiving radiotherapy with or without chemotherapy: a systematic literature review. Radiother Oncol 2003; 66:253-262.

9 Trotti A, et al: TAME: development of a new method for summarising adverse events of cancer treatment by the Radiation Therapy Oncology Group. Lancet Oncol 2007;8:613-624.

10 Machtay M, et al: Factors associated with severe late toxicity after concurrent chemoradiation for locally advanced head and neck cancer: an RTOG analysis. J Clin Oncol 2008;26:3582-3589.

11 Wilson JA, Carding PN, Patterson JM: Dysphagia after nonsurgical head and neck cancer treatment: patients' perspectives. Otolaryngol Head Neck Surg 2011;145:767-771.

12 Moor JW, et al: Prophylactic gastrostomy before chemoradiation in advanced head and neck cancer: a multiprofessional web-based survey to identify current practice and to analyse decision making. Clin Oncol (R Coll Radiol) 2010;22:192-198.

13 Paleri V, Patterson J: Use of gastrostomy in head and neck cancer: a systematic review to identify areas for future research. Clin Otolaryngol 2010;35:177-189.

14 Nutting CM, et al: Parotid-sparing intensity modulated versus conventional radiotherapy in head and neck cancer (PARSPORT): a phase 3 multicentre randomised controlled trial. Lancet Oncol 2011;12:127-136.

15 Hamilton DW, et al: Quality compared to quantity of life in laryngeal cancer: a time trade-off study. Head Neck 2016;38(suppl 1):E631-E637.

16 Ling DC, et al: Oncologic outcomes and patient-reported quality of life in patients with oropharyngeal squamous cell carcinoma treated with definitive transoral robotic surgery versus definitive chemoradiation. Oral Oncol 2016;61:41-46.

17 Sharma A, et al: Survival and gastrostomy prevalence in patients with oropharyngeal cancer treated with transoral robotic surgery vs chemoradiotherapy. JAMA Otolaryngol Head Neck Surg 2016;142:691-697.

18 Gildener-Leapman N, et al: Utility of up-front transoral robotic surgery in tailoring adjuvant therapy. Head Neck 2016;38:1201-1207.

19 De Almeida JR, et al: Oncologic outcomes after transoral robotic surgery: a multi-institutional study. JAMA Otolaryngol Head Neck Surg 2015;141:1043-1051.

20 Cohen MA, et al: Transoral robotic surgery and human papillomavirus status: oncologic results. Head Neck 2011;33:573-580.

21 Moore EJ, et al: Long-term functional and oncologic results of transoral robotic surgery for oropharyngeal squamous cell carcinoma. Mayo Clin Proc 2012;87:219-225.

22 Sload R, et al: The role of transoral robotic surgery in the management of HPV negative oropharyngeal squamous cell carcinoma. Curr Oncol Rep 2016;18:53.

23 Health and Social Care Information Centre: National Head and Neck Cancer Audit 2014 - key findings for England and Wales for the audit period November 2013 to October 2014. 2014.

24 Villanueva NL, et al: Transoral robotic surgery for the management of oropharyngeal minor salivary gland tumors. Head Neck 2014;36:28-33.

25 Hinni ML, et al: Surgical margins in head and neck cancer: a contemporary review. Head Neck 2013;35:13621370.

26 Huet PC: L'électro-coagulation dans l'épithelioma de l'amygdale-palatine. Ann Otolaryngol Chir Cervicofac 1951;68:433-442.

27 Holsinger FC, et al: Transoral lateral oropharyngectomy for squamous cell carcinoma of the tonsillar region. I. Technique, complications, and functional results. Arch Otolaryngol Head Neck Surg 2005;131:583-591.

28 Weinstein GS, et al: Transoral robotic surgery: radical tonsillectomy. Arch Otolaryngol Head Neck Surg 2007; 133:1220-1226.

29 De Almeida JR, et al: A systematic review of transoral robotic surgery and radiotherapy for early oropharynx cancer: a systematic review. Laryngoscope 2014;124:2096-2102.

30 Owen S, et al: Baseline swallowing measures predict recovery at 6 weeks after transoral robotic surgery for head and neck cancer. Clin Otolaryngol 2017;42:366-372. 
Paleri et al.: TORS for Oropharyngeal Cancer

31 Mandal R, et al: Analysis of post-transoral robotic-assisted surgery hemorrhage: frequency, outcomes, and prevention. Head Neck 2016;38(suppl 1):E776-E782.

32 Pollei TR, et al: Analysis of postoperative bleeding and risk factors in transoral surgery of the oropharynx. JAMA Otolaryngol Head Neck Surg 2013;139:1212-1218.

33 Bernier J, et al: Defining risk levels in locally advanced head and neck cancers: a comparative analysis of concurrent postoperative radiation plus chemotherapy trials of the EORTC (\# 22931) and RTOG (\# 9501). Head Neck 2005;27:843-850.

34 Garden AS, et al: Radiation therapy (with or without neck surgery) for phenotypic human papillomavirusassociated oropharyngeal cancer. Cancer 2016;122:1702-1707.

35 Wilkie MD, et al: Transoral laser microsurgery for oropharyngeal squamous cell carcinoma: a paradigm shift in therapeutic approach. Head Neck 2016;38:1263-1270.

36 Mehta V, et al: A new paradigm for the diagnosis and management of unknown primary tumors of the head and neck: a role for transoral robotic surgery. Laryngoscope 2013;123:146-151.

37 Fu TS, et al: The role of transoral robotic surgery, transoral laser microsurgery, and lingual tonsillectomy in the identification of head and neck squamous cell carcinoma of unknown primary origin: a systematic review. J Otolaryngol Head Neck Surg 2016;45:28.

38 Winter SC, et al: Transoral robotic assisted tongue base mucosectomy for investigation of unknown primary in the head and neck region: the UK experience. Clin Otolaryngol 2017;42:1247-1251.

39 Su HK, et al: Safety of transoral surgery for oropharyngeal malignancies: an analysis of the ACS NSQIP. Laryngoscope 2016;126:2484-2491.

40 Paleri V, et al: Transoral robotic surgery for residual and recurrent oropharyngeal cancers: an IDEAL phase 2a exploratory study of surgical innovation. Head Neck 2018;40:512-525.

41 De Almeida JR, et al: Reconstructive algorithm and classification system for transoral oropharyngeal defects. Head Neck 2014;36:934-941.

42 Longfield EA, Holsinger FC, Selber JC: Reconstruction after robotic head and neck surgery: when and why. J Reconstr Microsurg 2012;28:445-450.

43 White H, et al: Salvage surgery for recurrent cancers of the oropharynx: comparing TORS with standard open surgical approaches. JAMA Otolaryngol Head Neck Surg 2013;139:773-778.

44 Dabas S, et al: Salvage transoral robotic surgery for recurrent or residual head and neck squamous cell carcinoma: a single institution experience. Asian Pac J Cancer Prev 2015;16:7627-7632.

45 Ang KK, Harris J, Wheeler R, Weber R, Rosenthal DI, Nguyen-Tân PF, et al: Human papillomavirus and survival of patients with oropharyngeal cancer. N Engl J Med 2010;363:24-35. 